City University of New York (CUNY) CUNY Academic Works

\title{
Distributed Cross-Community Collaboration for the Cloud-Based Energy Management Service
}

Yu-Wen Chen

CUNY New York City College of Technology

J. Morris Chang

University of South Florida

\section{How does access to this work benefit you? Let us know!}

More information about this work at: https://academicworks.cuny.edu/ny_pubs/825

Discover additional works at: https://academicworks.cuny.edu

This work is made publicly available by the City University of New York (CUNY).

Contact: AcademicWorks@cuny.edu 


\section{Distributed Cross-Community Collaboration for the Cloud-Based Energy Management Service}

\author{
Yu-Wen Chen \\ Computer Systems Technology \\ New York City College of Technology \\ Brooklyn, NY, USA \\ e-mail: YWChen@citytech.cuny.edu
}

\author{
J. Morris Chang \\ Electrical Engineering \\ University of South Florida \\ Tampa, FL, USA \\ e-mail: chang5@usf.edu
}

\begin{abstract}
Customers' participation is a critical factor for integrating the distributed energy resources via demand response and demand-side management programs, especially when customers become prosumers. Incentives need to be delivered by the energy management service to attract prosumers to operate their distributed energy resources and electricity loads grid-friendly actively. The cloud-based energy management service enables virtual trading for customers within the same community to minimize cost and smooth the fluctuation. With the potential fast-growing number of service providers and customers, the needs exist for efficiently collaborating across multiple service providers and customers. This paper proposes the distributed cross-community collaboration (XCC) for the cloud-based energy management service to enable collaboration across multiple communities and service providers. The XCC can efficiently handle large-scale variables and data with various allocated computing resources and is formulated as an alternating direction method of multipliers optimization problem. This paper also introduces a cross-community adjustment to avoid the overwhelmed exchanged data and computations among multiple communities under uncertainty. Performances are evaluated in experiments with the discussions.
\end{abstract}

Keywords-distributed large scale, cloud-based, energy management service, EMaaS, cross-community, smart grid, distributed energy resources, trading choices, ADMM

\section{NOMENCLATURE}

\section{Parameters:}

$G$ DER production capacity.

$T^{c}$ Assigned capacity for power distribution line.

$P^{b}$ Price for buying energy from power grid to community.

$P^{s} \quad$ Price for selling produced renewable energy from community to power grid.

$P^{r}$ Price for trading within each community.

$D$ Summation of the requested fix loads.

$\eta c \quad$ Charging efficiency for storage.

$\eta d$ Discharging efficiency for storage.

$\gamma s$ (Dis)charging rate of storage.

$T^{u}$ Upper bound for smoothing the fluctuation.

$T^{l} \quad$ Lower bound for smoothing the fluctuation.

\section{Subscripts:}

$m \quad m_{t h}$ community.

$i \quad i_{\text {th }}$ customer.

$j \quad j_{t h}$ interruptible or non-interruptible load. $t \quad t_{t h}$ time step.

$z \quad z_{t h}$ power distribution line.

Sets:

(1) For the involved community.

$\mathbb{N}$ For customers within each community.

$\mathbb{T}$ For time step, from 1 to $t^{e}$.

$\mathbb{Z} \quad$ For power distribution lines.

$\mathbb{L}$ For customers connected to the same power distribution line.

Choice variables for DER:

$E^{r c}$ Export produced energy from DER to community.

$E^{r m}$ Export produced energy from DER to power grid.

$E^{r s}$ Export produced energy from DER to storage.

$E^{r d}$ Export produced energy from DER for load usage.

Choice variables for storage:

$I^{m s}$ Import energy from power grid to storage.

$I^{c s}$ Import energy from community to storage.

$I^{r s}$ Import energy from DER to storage.

$E^{s m}$ Export energy from storage to power grid.

$E^{s c}$ Export energy from storage to community.

$E^{s d}$ Export energy from storage for load usage.

$S \quad$ State of charge for storage.

Choice variables for loads:

$I^{m d}$ Use the energy from power grid.

$I^{c d}$ Use the energy from community grid.

$I^{r d}$ Use the energy from DER.

$I^{s d}$ Use the energy from storage.

\section{INTRODUCTION}

The active consumers' participation is listed as one of the most important characteristics of a Smart Grid [1]. Especially when customers have transformed from consumer to prosumers by adopting distributed energy resources (DERs), engaging customers with the proper management while encouraging the investment of DERs to promote the environmentfriendly power grid becomes a critical and essential mission. Besides, the widely discussed Demand Response (DR) and Demand Side Management (DSM) programs rely on customers' engagements as the opportunities are provided for customers changing and managing their "electrical usage choice." That is, adjusting their consumption pattern according 
to the fluctuated electricity prices. Thus, how to provide incentives to customers for encouraging them to involve energy management is an important issue.

Varieties of DR and DSM programs [2] have been developed for providing incentives to utilities and customers in literature. Authors in [3] applied the Stackelberg game among multiple utility companies and consumers. However, different from the cloud-based framework, difficulties exist in the above literature as the requirements of deploying the dedicated control entity and implementing the corresponding control mechanisms repeatedly for adoption by various utilities and customers. Along with the literature in DR and DSM programs, net metering [4] are broadly mentioned to realize the trading between customers and utilities. Still, only a few retail electricity providers (REP) [5] provide the excess generation buy-back program, such as Green Mountain Energy [6]. The incentives for customers' engagements are still insufficient as the trading choices are limited for prosumers. If prosumers can collaborate, their global costs can be reduced as the increased incentives via the trading among each other.

To address the above-mentioned difficulties and limitations, our previous work [7] proposed an extensive cloud-based framework to provide customer-oriented Energy Management as a Service (EMaaS) for "community", which is formed by prosumers who agree to involve in the same EMaaS plan. The EMaaS is provided by the EMaaS providers and is similar to the REP's existing energy service plan. A new price indicator appears when the community is formed and is agreed upon among all the customers. It is utilized by the customers to perform "virtual trading" within the community. This trading is virtual as the physical two-way power distribution line might not exist among customers and can be realized efficiently through a mapping mechanism on the cloud-based framework. EMaaS successfully provides incentives to customers to involve energy management and also enhance renewable energy integration. With EMaaS, customers can form virtual REPs and have more "trading choices". Infrastructure costs are significantly reduced, and efficiency, reliability, and scalability are increased. The choices of electricity usage and trading are combined to realize the fair DR with EV on the cloudbased energy management in [8]. Although our previous two works have managed a significant amount of variables, yet the choices are still limited within the same community, and the centralized computing resources maintain the management service. When a collaboration scheme for multiple communities and service providers exists, more "choices" will appear for customers to adjust their operation behavior to achieve the lower global cost, which is the incentive to promote the prosumers' participation. The collaboration scheme can also coordinate the distributed energy resources across multiple communities as a larger unit to support the integration.

This paper proposed cross-community collaboration (XCC) for the cloud-based energy management service to realize the collaborations among communities. XCC is developed in a distributed fashion to efficiently handle the large-scale data and variables with various allocated cloud computing resources.
We observed similar characteristics to the sharing problem [9] and formulated the XCC as a multi-block alternating direction method of multipliers (ADMM) [10] problem. The ADMM has been popularly used in various areas such as machine learning, data mining [11], and successfully applied to various power system tasks. Authors in [12] formulated the optimal power scheduling as an ADMM problem. Authors in [13] proposed the EV charging ADMM framework to perform the optimal fleet charging. A distributed demand response strategy with EV was proposed in [9], and [14] proposed the decentralized economic dispatch using ADMM. Authors in [15] adopted the ADMM for the multi-agent optimization problem. Unlike the above-discussed literatures, the ADMM model for the proposed distributed XCC is more complicated due to the large-scale of the emerged choices among customers and various communities.

The proposed XCC considers the global costs, which include the cost of electricity and environment, are minimized as the incentives are maximized for customers not only within the same community but also in all the collaborated communities and service providers. It is a collaborative business model for prosumers in the distributed power system. Depending on the utilized various cloud service providers, the distributed XCC for the cloud-based energy management service is performed either on the inter-cloud or the intra-cloud level [16], where the communication time in these two levels is small [17]. To avoid the overwhelmed exchanged data and computations among multiple communities under uncertainty, we also proposed a cross-community adjustment (XCA) process to prevent the scenarios where each community could frequently rerun the EMaaS according to the adjusting process [7].

The contributions of this paper are summarized below. (i) This work proposed cross-community collaboration (XCC) for the cloud-based energy management service. Incentives can deliver to customers to engage their participation by minimizing the global costs within all the cooperated communities and various service providers over the given time period. (ii) The energy management service with the XCC is formulated as the multi-blocks ADMM model to efficiently handle the large-scale data and variables with various allocated computing resources. The cross-community adjustment is introduced to avoid the overwhelmed exchanged data and computations among multiple communities under uncertainty. Performances are evaluated with different experiments along with a centralized model. The remainder of this paper is organized as follows: the system model is introduced in Section II. Formulations are presented in Section III. Performance evaluation is discussed in Section IV, and conclusions are summarized in Section V.

\section{SYSTEM MODEL}

To introduce the proposed system model, we break down the framework, the procedure for a single community, the model for a single community, the cross-community collaboration model, the cross-community adjustment, and the collaborative procedure in the following subsections. Throughout the paper, 
the subscription of $\{m, i, j, t, z\}$ are used to refer the $m_{t h}$ community, $i_{t h}$ customer, $j_{t h}$ interruptible or non-interruptible load, $t_{t h}$ time step and $z_{t h}$ power distribution line. The sets of $\{\mathbb{O}, \mathbb{N}, \mathbb{T}, \mathbb{Z}, \mathbb{L}\}$ are used to represent for the involved community, for customers within each community, for time step from 1 to $t^{e}$, for the power distribution lines, and for customers connected to the same power distribution line. For the simplification, the discussed number of multiple communities is set to three, which are indicated as $\{c o m m 1, \operatorname{comm} 2$, comm 3$\}$ in the set $\mathbb{O}$ throughout the paper.

\section{A. Framework}

The framework for the cloud-based energy management with cross-community collaboration (XCC) and crosscommunity adjustment (XCA) is illustrated in Fig. 1. It is constructed by multiple energy management service providers, a power grid, conventional power companies, and multiple communities form by multiple customers. Different energy management service providers operate the energy management on the cloud with different allocated computing resources and provide various service plans to communities via the thin client interfaces, e.g. web browsers or application programming interface (API). These service plans are similar to the various plans provided by existing REPs, where customers can choose to join by agreeing on the contract's requirements to each energy management service provider. The energy management service providers realize the XCC and XCA by collaborating via the XCC manager. The XCC manager can be maintained by the distributed system operator or the union of the group of service providers. Each customer represents various household sizes and forms the community with other customers who also choose the same service plan. The virtual trading is performed within each community with the dot lines and across multiple communities with the dash lines.

Conventional power companies and the DERs supply the power grid within each community following the interconnection agreement with local electric transmission and distribution utilities [18]. Customers are assumed to own a small-scale nondispatchable DER and a storage system in this work. Each DER has a set of choices variables $\left\{E^{r c}, E^{r m}, E^{r s}, E^{r d}\right\}$, and various production capacity $G$. The storage system can be powered by both DERs and the conventional generators with the (dis)charging efficiency rate $\{\eta c, \eta d\}$, and has the maximum/minimum storage capacity $\left\{S^{\max }, S^{\min }\right\}$. Each storage system is assumed can be store and release energy quickly and has a set of choice variables $\left\{I^{m s}, I^{c s}, I^{r s}, E^{s m}, E^{s c}, E^{s d}\right\}$.

\section{B. Procedure for Single Community}

The procedure for the single community is shown in Fig. 2. The energy management service provider gathers the $K$ time steps ahead data on the cloud, which includes $\left\{G, T^{c}, P^{b}, P^{s}, P^{r}, D\right\}$ and all the parameters of each storage system. They are the know inputs for the energy management service, and can be acquired via forecast technics $\left(G, P^{b}, P^{s}\right)$, customers' direct inputs ( $D$, parameters of the storage system), and the agreed contracts between customers and service

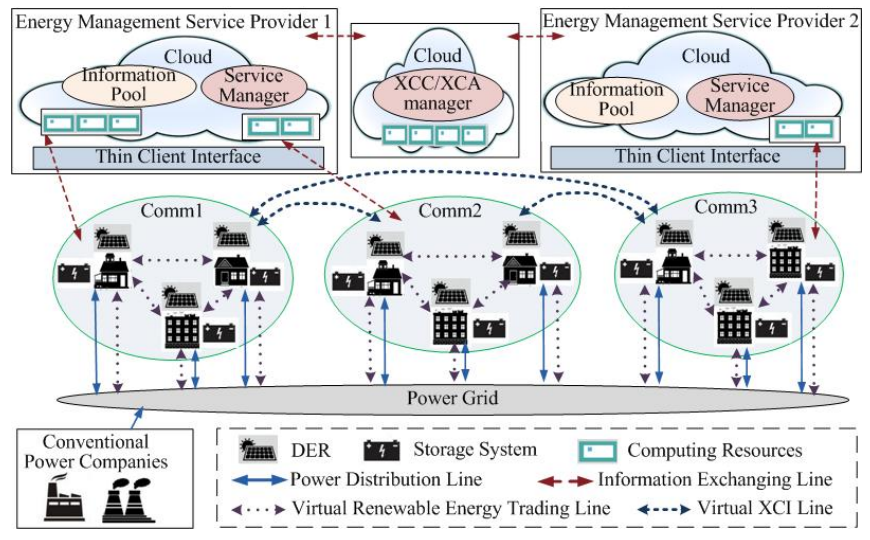

Fig. 1. Framework for the energy management service with XCC and XCA

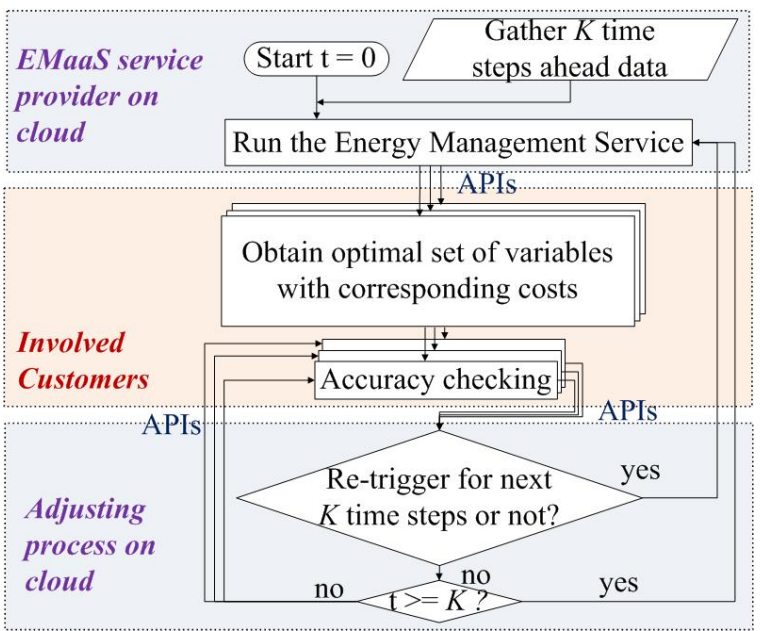

Fig. 2. Procedure for single community

providers $\left(P^{r}, T^{c}\right) \cdot P^{b}$ is the price for the customer buying the energy that is generated by the conventional power companies from the power grid. $P^{s}$ is the price for customers selling the produced renewable energy back to the power grid, and it is a smaller value than $P^{b}$ as it excludes the environment cost (i.e., $\mathrm{CO}_{2}$ emission cost or tax-saving provided by the government). $P^{r}$ is the price for customers trading to others within the same community. It is appeared and agreed by all the customers involved in the same service plan. The relationships among these three prices are shown in (1) and (2). $T^{c}$ is the assigned capacity for each power distribution line by local utilities and depends on the physical distribution network that supports both customers and non-customers of energy management service.

$$
\begin{aligned}
& P^{s}=\alpha P^{b}, \quad 0<\alpha<1 \\
& P^{r}=\beta P^{b}, \quad \alpha<\beta<1
\end{aligned}
$$

With all these $K$ time steps ahead data, the energy management service provider runs the energy management on the cloud and provides the optimal set of variables with the corresponding cost as suggestions to all involved customers via APIs. The mapping mechanism is used to realize the virtual trading within the community, where customers follow the 
mapped amount to (dis)charge their storage system in (3) and send the energy request in (4) to the power grid.

$$
\begin{aligned}
\operatorname{Map}_{i, t}^{s}= & I_{i, t}^{c s}-\eta d^{s} E_{i, t}^{s c}-\eta d^{s} E_{i, t}^{s m}, \forall i \in \mathbb{N}, \forall t \in \mathbb{T} \\
\operatorname{Map}_{i, t}^{d}= & I_{i, t}^{m d}+I_{i, t}^{c d}+I_{i, t}^{m s}+I_{i, t}^{c s}-E_{i, t}^{r m}-E_{i, t}^{r c} \\
& -\eta d^{s} E_{i, t}^{s c}-\eta d^{s} E_{i, t}^{s m} \quad, \forall i \in \mathbb{N}, \forall t \in \mathbb{T} .
\end{aligned}
$$

The accuracy checking and the adjusting process in Fig. 2 are proposed to manage the uncertainties (e.g., sudden electricity usage changes or the operations not following the suggestions) and the forecast errors. The accuracy checking part informs the real-time variance to the adjusting process, and the adjusting process determines to re-trigger the next $K$ time steps ahead management or not. The complete information of the adjusting process can be found from the work [7].

\section{Model for Single Community}

The energy management service for a single community can be formulated as a linear programming model with the 14 choice variables and a state variable $S$ for each customer at each time step. The objective function $f(x)$ is minimizing the electricity cost over $K$ time steps, as shown in (5). It is the summation of each customer's cost of fulfilling the demands $\left(C_{o s t}^{d}\right)$, trading within the community $\left(\right.$ Cost $\left.^{r}\right)$, and managing the storage system $\left(\operatorname{Cost}^{s}\right)$.

$$
\begin{aligned}
& \min f(x)=\sum_{t \in \mathbb{T}}\left(\sum_{i \in \mathbb{N}}\left(\operatorname{Cost}_{i, t}^{d}+\operatorname{Cost}_{i, t}^{r}+\operatorname{Cost}_{i, t}^{s}\right)\right) \\
& \operatorname{Cost}_{i, t}^{d}=I_{i, t}^{m d} P_{t}^{b}+I_{i, t}^{c d} P_{i, t}^{r b} \\
& \operatorname{Cost}_{i, t}^{r}=-E_{i, t}^{r m} P_{t}^{s}-E_{i, t}^{r c} P_{i, t}^{r s} \\
& \operatorname{Cost}_{i, t}^{s}=I_{i, t}^{m s} P_{t}^{b}+I_{i, t}^{c s} P_{i, t}^{r b}-\eta d^{s}\left(E_{i, t}^{s m} P_{t}^{s}+E_{i, t}^{s c} P_{i, t}^{r s}\right)
\end{aligned}
$$

The objective function subjects to constraints (9) to (23). Constraint (9) ensures the customer's demand can be satisfied, and (10) guarantees the mapped electricity requests in (4) from customers located on the same $z_{t h}$ power distribution line won't exceed the assigned capacity. Constraint (11) prevents the exported amount of energy from each DER exceed its production capacity, and (12) forbids customer from exporting more than the imported amount of energy from the community. The total available energy for trading within the community is tracked in (13) to avert customers request more than the available amount. For the storage system, (14) indicates its status depends on the previous status and the variables at the current time step. Constraints (15) and (16) ensure the storage is operated in its maximum and minimum capacity after exporting energy at every time steps. Constraint (17) is similar to (12) to prevent the storage exporting the energy to the community more than the imported amount. The (dis)charging rate and the efficiency constrain the variables in (18)-(19). Constraints (20)-(22) indicate the corresponding variables for internal behavior-changing have to be matched. Constraint (23) bound the total imported amount of energy from the power grid with $T^{u}$ and $T^{l}$. These two parameters are utilized to smooth the fluctuation within the community and are agreed between EMaaS provider and the local utility.

$$
\begin{aligned}
& D_{i, t}=I_{i, t}^{m d}+I_{i, t}^{c d}+I_{i, t}^{r d}+I_{i, t}^{s d} \quad, \forall i, \forall t . \quad \text { (9) } \\
& \sum_{i \in \mathbb{L}_{z}} \operatorname{Map}_{i, t}^{d} \leq T_{z, t}^{c} \quad, \forall z, \forall t . \text { (10) } \\
& G_{i, t}=E_{i, t}^{r m}+E_{i, t}^{r c}+E_{i, t}^{r s}+E_{i, t}^{r d} \quad, \forall i, \forall t .(11) \\
& E_{i, t}^{r c}-I_{i, t}^{c d}-I_{i, t}^{c s} \leq 0 \\
& , \forall i, \forall t \text {. (12) } \\
& \sum_{i \in \mathbb{N}} \eta d^{s} E_{i, t}^{s c}-I_{i, t}^{c s}+E_{i, t}^{r c}-I_{i, t}^{c d}=0 \\
& S_{i, t+1}=S_{i, t}+\eta c^{s} \times\left(I_{i, t}^{m s}+I_{i, t}^{c s}+I_{i, t}^{r s}\right) \\
& -\left(E_{i, t}^{s m}+E_{i, t}^{s c}+E_{i, t}^{s d}\right) \\
& S_{i}^{\text {min }} \leq S_{i, t} \leq S_{i}^{\max } \\
& S_{i, t}-E_{i, t}^{s m}-E_{i, t}^{s c}-E_{i, t}^{s d} \geq S_{i}^{m i n} \\
& \sum_{t^{\prime}=1}^{t-1}\left(\eta^{s c} I_{i, t^{\prime}}^{r s}+\eta^{s c} I_{i, t^{\prime}}^{c s}-E_{i, t^{\prime}}^{s c}\right)-E_{i, t}^{s c} \geq 0 \\
& \eta c^{e} \times\left(I_{i, t}^{m s}+I_{i, t}^{c s}+I_{i, t}^{r s}\right) \leq \gamma_{i}^{s} \\
& \eta d^{e} \times\left(E_{i, t}^{s m}+E_{i, t}^{s c}+E_{i, t}^{s d}\right) \leq \gamma_{i}^{s} \\
& I_{i, t}^{r d}-E_{i, t}^{r d}=0 \\
& I_{i, t}^{r s}-E_{i, t}^{r s}=0 \\
& I_{i, t}^{s d}-\eta d_{i}^{s} E_{i, t}^{s d}=0 \\
& T_{t}^{l} \leq \sum_{i \in \mathbb{N}}\left\{I_{i, t}^{m d}+I_{i, t}^{m s}-E_{i, t}^{r m}-\eta d^{s} E_{i, t}^{s m}\right\} \leq T_{t}^{u}, \forall t .
\end{aligned}
$$

The model can be written in the matrix format with the vector sets, $\boldsymbol{x}$. The size of each element of $\boldsymbol{x}$ is $n^{x}$ that represents every customers' 15 variables at each time step, i.e. $\boldsymbol{x}=\left[\boldsymbol{x}_{1}, \boldsymbol{x}_{2}, \cdots, \boldsymbol{x}_{t^{e}}\right]$. Each element is arranged follows the order of variables in (24) from the first customer to the last customer within the community.

$$
\begin{aligned}
& \boldsymbol{x}_{1}=\left[\left[I_{i, t}^{m d}, I_{i, t}^{m s}, E_{i, t}^{r m}, E_{i, t}^{s m}, I_{i, t}^{c d}, I_{i, t}^{r d}, I_{i, t}^{s d}, E_{i, t}^{r c}, E_{i, t}^{r s}, E_{i, t}^{r d},\right.\right. \\
& \left.\left.I_{i, t}^{c s}, I_{i, t}^{r s}, E_{i, t}^{s c}, E_{i, t}^{s d}, S\right]_{i=1},[\cdots]_{i=2}, \cdots\right]_{t=1, \forall i}
\end{aligned}
$$

The objective function, (5)-(8), becomes (25), where $\boldsymbol{O} \boldsymbol{b} \boldsymbol{j}$ is the objective matrix indicating the constant coefficients in (6)(8). The equality constraints are rewritten as $\boldsymbol{P}^{e q}$ and $\boldsymbol{b e} \boldsymbol{q}$ in (27). Likewise, the rest inequality constraints are transformed into $\boldsymbol{P}^{i e q}$ and $\boldsymbol{b}$ in (26).

$$
\begin{array}{ll}
\min & f(\boldsymbol{x})=\boldsymbol{O} \boldsymbol{b} \boldsymbol{j} \times \boldsymbol{x} \\
\text { subject to: } & \boldsymbol{P}^{i e q} \boldsymbol{x} \geq \boldsymbol{b} \\
& \boldsymbol{P}^{e q} \boldsymbol{x}=\boldsymbol{b} \boldsymbol{e} \boldsymbol{q}
\end{array}
$$

\section{Model for Cross-community Collaboration}

A vector $z$ is introduced to interpret the trading behaviors among the involved communities. Each element in the vector $z$ represents two corresponding behaviors between any two of the involved communities. The size of $z, n^{z}$, depends on the number of the involved communities $\left(n^{c}\right)$, which is $n^{c} \times\left(n^{c}-\right.$ 1 ). With 3 as the discussed size of the involved community in this paper, the size of $z$ is 6 (i.e., $z^{1}, \cdots, z^{6}$ ). The represented behaviors for each element in $z$ are listed in Table I. 
TABLE I

PRESENTATION OF $z$ FOR 3 INVOLVED COMMUNITIES

\begin{tabular}{|c|c|c|}
\hline Variables & Interaction behaviors & Constrains \\
\hline$z^{1}$ & $\begin{array}{l}\operatorname{comm} 1 \text { buy from } \operatorname{comm} 2 \text {; } \\
\text { comm } 2 \text { sell to } \operatorname{comm} 1\end{array}$ & $\leq l_{1}$ \\
\hline$z^{2}$ & $\begin{array}{l}\text { comm } 1 \text { buy from comm } 3 \\
\text { comm } 3 \text { sell to comm } 1\end{array}$ & $\leq l_{2}$ \\
\hline$z^{3}$ & $\begin{array}{l}\text { comm } 2 \text { buy from } \operatorname{comm} 1 \\
\text { comm } 1 \text { sell to comm } 2\end{array}$ & $\leq l_{1}$ \\
\hline$z^{4}$ & $\begin{array}{l}\text { comm2 buy from comm } 3 \\
\text { comm } 3 \text { sell to comm } 2\end{array}$ & $\leq l_{3}$ \\
\hline$z^{5}$ & $\begin{array}{l}\text { comm } 3 \text { buy from comm } 1 \\
\text { comm } 1 \text { sell to comm } 3\end{array}$ & $\leq l_{2}$ \\
\hline$z^{6}$ & $\begin{array}{l}\text { comm } 3 \text { buy from comm } 2 \\
\text { comm } 2 \text { sell to comm } 3\end{array}$ & $\leq l_{3}$ \\
\hline
\end{tabular}

The objective function $g(\boldsymbol{z})$ for the XCC is for each community to minimize the cost via the trading across each communities, as shown in (28). The community that buys from other communities can reduce the cost with the trading amount times its purchasing price from the main power grid, $P^{b}$. Likewise, the community that sells to other communities can increase the cost with the trading amount times its selling price to the main power grid, $P^{s}$.

$$
\begin{aligned}
& g(\boldsymbol{z})=\left(P_{\text {comm } 2}^{s}-P_{\text {comm } 1}^{b}\right) z^{1}+\left(P_{\text {comm } 3}^{s}-P_{\text {comm } 1}^{b}\right) z^{2} \\
& \quad+\left(P_{\text {comm } 1}^{s}-P_{\text {comm } 2}^{b}\right) z^{3}+\left(P_{\text {comm } 3}^{s}-P_{\text {comm } 2}^{b}\right) z^{4} \\
& \quad+\left(P_{\text {comm } 1}^{s}-P_{\text {comm } 3}^{b}\right) z^{5}+\left(P_{\text {comm } 2}^{s}-P_{\text {comm } 3}^{b}\right) z^{6}
\end{aligned}
$$

Each element of $z$ is constrained by the corresponding community's exporting/importing amount to/from the main power grid, that is $\left(E^{r m}+E^{s m}\right)$ and $\left(I^{m d}+I^{m s}\right)$. The element of $\boldsymbol{z}$ is also constrained by the assigned available line capacity between every two communities as listed in the last column in Table I, where $\left\{l_{1}, l_{2}, l_{3}\right\}$ denote the available line capacity between (comm 1, comm 2$)$, (comm 1, comm 3 ) and (comm2, comm 3$)$ respectively. The interaction behavior constraints for each element of $z$ can be rewritten along with the vector $\boldsymbol{x}$ in (29) for each community in each time step. Likewise, the constraints for the available line capacity in Table I can be written in (30).

$$
\begin{aligned}
& \boldsymbol{A} \boldsymbol{x} \geq \boldsymbol{D} \boldsymbol{z} \\
& \mathbb{1} \boldsymbol{z} \leq \boldsymbol{l}
\end{aligned}
$$

\section{E. Cross-community Adjustment and Collaborative Procedure}

The adjustment process in Fig. 2 is maintained by each community individually. Without proper management scheme, the individual adjustment process in each community might cause the overwhelmed computations and exchanged data. The cross-community adjustment (XCA) is proposed to collaborate with the communities who physically located on the same electrical distribution line or region. The XCA is maintained by the XCA manager, which served by the same organization that serve the XCC manager.

The collaborative procedure with the XCC and XCA is presented in Fig. 3. The XCC manager gathers the updated data from all involved communities and run the energy

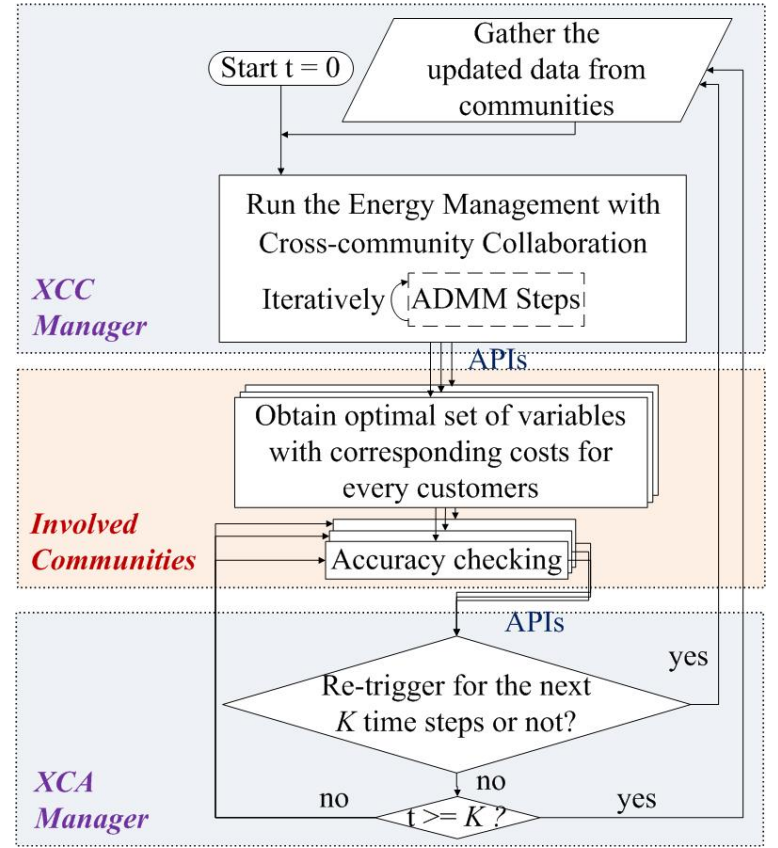

Fig. 3. Collaborative Procedure with XCC and XCA

management with the cross-community collaboration via the iterative ADMM steps, which is introduced in details in subsection III-B. The optimal set of variables are delivered to every customers within all the involved communities. For communities that are physically located on the same electrical distribution line or region will then forward the accuracy checking output to the XCA manager.

\section{FORMULATION}

\section{A. Centralized problem}

Combining the models in subsection II-C and II-D, the energy management service with the XCC for three involved communities can be formulated as a centralized optimization problem, where the objective function (31) that subjects to constraints (32)-(34).

$$
\min _{\boldsymbol{x}_{m}, \boldsymbol{z}} \sum_{m \in \mathbb{O}} f_{m}\left(\boldsymbol{x}_{m}\right)+g(\boldsymbol{z})
$$

subject to: (30), and

$$
\begin{aligned}
& \boldsymbol{A}_{m} \boldsymbol{x}_{m} \geq \boldsymbol{D}_{m} \boldsymbol{z}, \forall m \in \mathbb{O} \\
& \boldsymbol{P}_{m}^{i e q} \boldsymbol{x}_{m} \geq \boldsymbol{b}_{m}, \forall m \in \mathbb{O} \\
& \boldsymbol{P}_{m}^{e q} \boldsymbol{x}_{m}=\boldsymbol{b e q}_{m}, \forall m \in \mathbb{O}
\end{aligned}
$$

\section{B. Distributed multi-block ADMM problem}

The number of the variables and constraints grow significantly when the involved customers and the service providers increase, operating the service in the centralize approach would be inefficient and not practical. Thus, we formulated the problem as the alternating direction of multiplier method (ADMM) [10] problem due to the similar characteristics of the standard sharing problem [9]. The process is discussed below. 
First, we decouple the global variable $z$ in the objective function (31) into $z_{m}$ for each community and represent the objective function as (35). Constraint (37) is designed to ensure the decoupled $z_{m}$ achieve the same global variable $\phi$, where the indicated matrix $\boldsymbol{C}_{m}$ for the related $\boldsymbol{z}_{\boldsymbol{m}}$ within each community. A new variable $Y$ is created to convert the inequality constraint (32) to the equality one in (36).

$$
\min _{\boldsymbol{x}_{m}, \boldsymbol{z}_{m}, \boldsymbol{Y}_{m}, \boldsymbol{\phi}} \sum_{m \in \mathbb{O}}\left\{f_{m}\left(\boldsymbol{x}_{m}\right)+g\left(\boldsymbol{z}_{m}\right)\right\}
$$

subject to: (30), (33), (34), and

$$
\begin{aligned}
& \boldsymbol{A}_{m} \boldsymbol{x}_{m}=\boldsymbol{D}_{m} \mathbf{z}_{m}+\boldsymbol{Y}_{m}, \forall m \in \mathbb{O} \\
& \boldsymbol{z}_{m}=\boldsymbol{C}_{m} \boldsymbol{\phi}, \forall m \in \mathbb{O}
\end{aligned}
$$

The variable vectors, $\boldsymbol{x}$ and $\boldsymbol{z}$, are maintained by each community for every time steps from $t=1 \cdots t^{c}$, and can be grouped as another variable vector $\boldsymbol{X}$. The problem in equations (35)(37) can be reformulated as the multi-block ADMM problem is formulated as shown in (38)-(42), where $\boldsymbol{B}_{m}$ is the matrix with the $n^{x} \times n^{z}$ zeros matrix and an identity matrix with size $n^{z}$ for every time step $t$.

$$
\begin{array}{ll}
\min _{\boldsymbol{X}_{m}, \boldsymbol{Y}_{m}, \boldsymbol{\phi}} & \sum_{m \in \mathbb{O}} \theta\left(\boldsymbol{X}_{m}\right) \\
\text { subject to: } & \boldsymbol{A}_{m} \boldsymbol{X}_{m}-\boldsymbol{Y}_{m}=\mathbf{0}, \forall m \in \mathbb{O} \\
& \boldsymbol{B}_{m} \boldsymbol{X}_{m}-\boldsymbol{C}_{m} \boldsymbol{\phi}=\mathbf{0}, \forall m \in \mathbb{O} \\
& \boldsymbol{G c}_{m}^{i e q} \boldsymbol{X}_{m} \geq \boldsymbol{G} \boldsymbol{b}_{m} \quad, \forall m \in \mathbb{O} \\
& \boldsymbol{G c}_{m}^{e q} \boldsymbol{X}_{m} \geq \boldsymbol{G b e q}_{m}, \forall m \in \mathbb{O}
\end{array}
$$

The problem can be efficiently solved iteratively [10]with its augmented lagrangian $(\mathcal{L})$ in $(43)$. The $\left\{\boldsymbol{\lambda}_{(1, m)}, \boldsymbol{\lambda}_{(2, m)}\right\}$ are the dual variables, $\left\{\rho_{1}, \rho_{2}\right\}$ are the penalty parameters, and $\langle\cdot\rangle$ denotes the inner product.

$$
\begin{aligned}
& \mathcal{L}\left(\boldsymbol{X}_{m}, \boldsymbol{Y}_{m}, \boldsymbol{\phi}, \boldsymbol{\lambda}_{(1, m)}, \boldsymbol{\lambda}_{(2, m)}\right)=\sum_{m \in \mathbb{O}} \theta\left(X_{m}\right) \\
& +\frac{\rho_{1}}{2} \sum_{m \in \mathbb{O}}\left\|\boldsymbol{A}_{m} \boldsymbol{X}_{m}-\boldsymbol{Y}_{m}\right\|^{2}+\left\langle\boldsymbol{A}_{m} \boldsymbol{X}_{m}-\boldsymbol{Y}_{m}, \boldsymbol{\lambda}_{(1, m)}\right\rangle \\
& +\frac{\rho_{2}}{2} \sum_{m \in \mathbb{O}}\left\|\boldsymbol{B}_{m} \boldsymbol{X}_{m}-\boldsymbol{C}_{m} \boldsymbol{\phi}\right\|^{2} \\
& +\left\langle\boldsymbol{B}_{m} \boldsymbol{X}_{m}-\boldsymbol{C}_{m} \boldsymbol{\phi}, \boldsymbol{\lambda}_{(2, m)}\right\rangle
\end{aligned}
$$

The conceptual diagram for the ADMM steps is presented in Fig. 4, and the iterative solving procedure for the fiveblock ADMM problem is listed in Algorithm 1. Variables $\left\{\boldsymbol{X}_{m}, \boldsymbol{Y}_{m}, \boldsymbol{\phi}, \boldsymbol{\lambda}_{(1, m)}, \boldsymbol{\lambda}_{(2, m)}\right\}$ are updated iteratively on various cloud computing resources, where the superscript $k$ denote as the iteration number. Every community $m$ update the $X_{m}^{k}$ following the (44) that subjects the constraint (41) and (42), and $Y_{m}^{k}$ with the closed form solution. Then each community's cloud computing resources send the updated $X_{m}^{k+1}$ and $Y_{m}^{k+1}$ to the cross-community collaboration manager's cloud computing resources to update the global variable $\phi^{k}$, the dual variables $\lambda_{(1, m)}^{k}$, and $\lambda_{(2, m)}^{k}$. After the update, variables

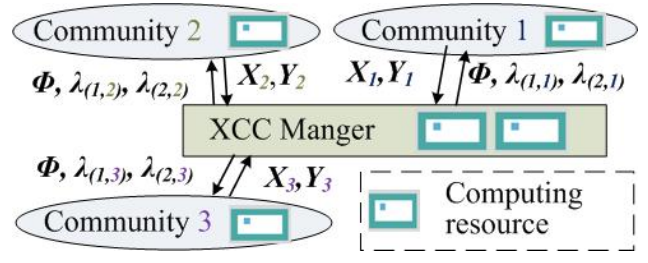

Fig. 4. Conceptual diagram for ADMM steps

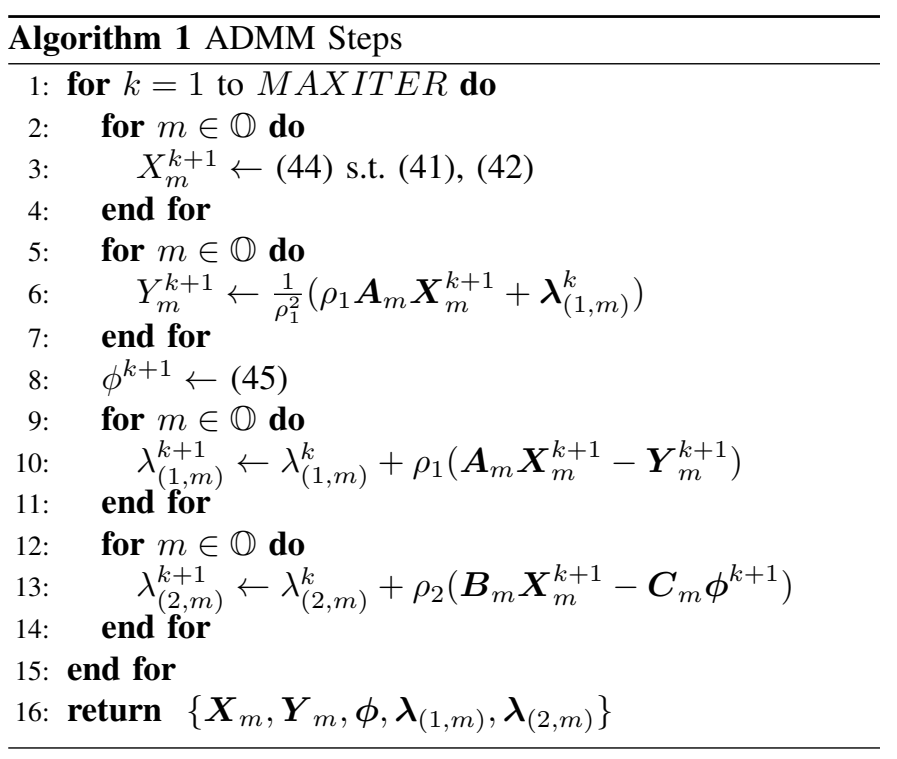

$\phi^{k+1}, \lambda_{(1, m)}^{k+1}, \lambda_{(2, m)}^{k+1}$ are sent back to each community's cloud computing resources.

$$
\begin{aligned}
\boldsymbol{X}_{m}^{k+1} & =\operatorname{argmin}_{\boldsymbol{X}_{m}}\left\{\theta\left(\boldsymbol{X}_{m}^{k}\right)+\frac{\rho_{1}}{2}\left\|\boldsymbol{A}_{m} \boldsymbol{X}_{m}^{k}-\boldsymbol{Y}_{m}^{k}\right\|^{2}\right. \\
& +\left\langle\boldsymbol{A}_{m} \boldsymbol{X}_{m}^{k}-\boldsymbol{Y}_{m}^{k}, \boldsymbol{\lambda}_{(1, m)}^{k}\right\rangle+\frac{\rho_{2}}{2}\left\|\boldsymbol{B}_{m} \boldsymbol{X}_{m}^{k}-\boldsymbol{C}_{m} \boldsymbol{\phi}^{k}\right\|^{2} \\
& \left.+\left\langle\boldsymbol{B}_{m} \boldsymbol{X}_{m}^{k}-\boldsymbol{C}_{m} \boldsymbol{\phi}^{k}, \boldsymbol{\lambda}_{(2, m)}^{k}\right\rangle\right\} \\
\boldsymbol{\phi}^{k+1} & =\operatorname{argmin}_{\boldsymbol{\phi}_{i}}\left\{\sum_{m \in \mathbb{O}} \theta\left(\boldsymbol{X}_{m}^{k+1}\right)\right. \\
& +\frac{\rho_{2}}{2} \sum_{m \in \mathbb{O}}\left\|\boldsymbol{B}_{m} \boldsymbol{X}_{m}^{k+1}-\boldsymbol{C}_{m} \boldsymbol{\phi}^{k}\right\|^{2} \\
& \left.+\left\langle\boldsymbol{B}_{m} \boldsymbol{X}_{m}^{k}-\boldsymbol{C}_{m} \boldsymbol{\phi}^{k}, \boldsymbol{\lambda}_{(2, m)}^{k}\right\rangle\right\}
\end{aligned}
$$

Convergence Analysis: The convergence of multi-block ADMM has been discussed widely in recently. Sufficient conditions have been established in [19] for K-block $(K \geq 3)$ ADMM. With the linear coupling constraints, our ADMM problem falls into the categorization in [20] and is guaranteed to converge to the global optimal solution.

\section{Performance Evaluation}

\section{A. Experiment Environment}

The time horizon $K$ is set to 24 by following the current existing day-ahead operation interval provided by CAISO [21], and each time step represents an hour. The production capacity of each customer's DER follows the $G$ basis in Fig. 5. According to the hourly day-ahead data from CAISO, solar 
TABLE II

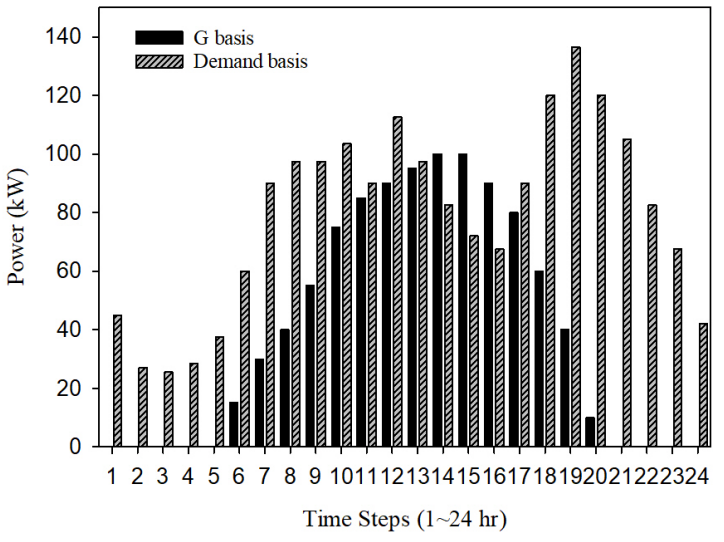

Fig. 5. Basis of demand \& renewable energy production

generations are average work from clock 6 to clock 20 a day during summer. To indicate the different DER configurations (i.e., size, angle or the location of shades, etc.), we multiply the basis to a uniform distribution, $\mathcal{U}(0.2,1.2)$ and $\mathcal{U}(0.8,1.8)$, to show the environments of low and high DER production capacity respectively. The requested demand for each customer is designed based on the typical household load profile (i.e., Demand basis in Fig. 5) times a uniform distribution $\mathcal{U}(0.5,1.5)$ to mimic the various sizes of households and customers' electricity usage preferences.

The discussed storage system follows the spec of Tesla Power Wall [22]. The $S^{\max }$ is $14 \mathrm{kWh}, \gamma s$ is $2 \mathrm{~kW}$, and both $\eta c, \eta d$ are 0.95 that match the power wall's round-trip efficiency. The storage capacity follows [23], where $S^{\text {min }}$ is $15 \%$ of $S^{\max }$. In this work, the blackout situation is assumed will not occur. Customers are designed to locate on different distribution lines in our experiment, and the available capacity of each power distribution line $\left(T^{c}\right)$ is assigned based on the number of connected customer times the largest requested electricity demand $D$. Our experiments set $T^{u}$ and $T^{l}$ as the unbounded values since the ability of the cloud-based energy management service to smooth the fluctuation within each community has been discussed in [7] and is not the focus in this paper. The prices indicators $\left\{P^{b}, P^{s}\right\}$ are acquired via the predictions and $P^{r}$ is agreed in contracts between the joined customers and each EMaaS service providers. To mimic the experiment more realistic, the design of $P^{b}$ for each community is based on the fuel cost predicted by a conventional generator. The conventional generator support 3000 households includes the involved customers and other households who didn't participate in the energy management service. The utilized quadratic fuel cost function in [24] $a+b(3000 \times A v g D e)+c(3000 \times A v g D e)^{2}$ sets the value of $P^{b}$ falls in the range of 1.4 to 9.8 cents per kWh. The AvgDe is the average of all customers' demands within the community and the coefficients $(a, b, c)=(240,7,0.007)$. The $\alpha$ and $\beta$ in (1) and (2) are designed as 0.4 and 0.7. The implementation is conducted in MATLAB.
Cost-SAVING PERFormANCE

\begin{tabular}{l||c|c|c||c|c|c}
\multicolumn{1}{l||}{} & \multicolumn{3}{c|}{ low DER production capacity } & \multicolumn{3}{c}{ high DER production capacity } \\
\hline Customers & 25 & 50 & 75 & 25 & 50 & 75 \\
\hline \hline$l=0$ & 2062.2 & 4867.2 & 7172.1 & 1467.0 & 3461.0 & 5237.4 \\
\hline$l=500$ & 1654.0 & 4398.8 & 6651.5 & 1017.2 & 2894.2 & 4621.9 \\
\hline$l=1000$ & 1387.5 & 4062.3 & 6267.9 & 685.33 & 2491.7 & 4142.7 \\
\hline$l=1500$ & 1208.7 & 3750.4 & 5936.8 & 391.42 & 2144.5 & 3748.5
\end{tabular}

\section{B. Cost-saving Performance}

The cost-saving performance is conducted with the experiments under the scenarios of high and low DER production capacity, the cases of $\{25,50,75\}$ customers in each community. The amount of the handled variables are significant large as the total number of the variables for each customers is $15 \times 24=360$. For the case with 50 customers in three communities, the total variables would be $(360 \times 50)^{3}$. The available community line capacities $(l)$ are set as $\{0,500,1000,1500$ $\mathrm{kW}$, where $l=0$ is the case without the cross-community collaboration. The results of the global cost with the unit as a dollar are listed in Table. II. The XCC for the cloudbased energy management service achieves a more significant advantage when the ratio of $l$ to the number of customers within each community is larger.

\section{Effects of the Storage System}

To present the effects of the storage system, Table. III shows the global cost with the unit as dollar for the cases with customer number as 25 in each community. With the storage system involved in energy management, the cost-saving can be further reduced. Moreover, the results also show that the proposed cross-community collaboration can reduce the cost when no storage system is involved. With the proposed crosscommunity collaboration, customers in the areas that lack storage systems can still acquire incentives to reduce their costs by getting involved in energy management.

\section{Converge Performance}

The converge performance is discussed with the simplified case, where each community has three customers. We also transformed the 24 hours data from 24-time steps into 4 indexes, where each index indicates the average amount within the original 6-time steps. Results are run under the low DER production capacity scenario, and the available line capacities $\left(l_{1}, l_{2}, l_{3}\right)$ are all set to 100 . Fig. 6 shows the convergence of the optimized objective value (hundred $\$ / k W h$ ) over the iterations with different settings of the penalty parameter $(\rho)$, which is set to equal to $\rho_{1}$, and $\rho_{2}$. The problem in (38) successfully converges regardless of the setting of $\rho$. To further show the difference affects from the various $\rho$, iteration from 25 to 150 are extracted in Fig. 7. When the $\rho$ is set to a larger value, the convergence rate is slower.

\section{E. Advantage of Cross-community adjustment}

To present the advantage of the cross-community adjustment (XCA), another program is implemented to mimic the example 
TABLE III

COST-SAVING PERFORMANCE WITH AND WITHOUT STORAGE SYSTEM

\begin{tabular}{c||c|c|c|c||c|c|c|c}
\multicolumn{1}{c||}{} & \multicolumn{4}{c||}{ low DER production capacity } & \multicolumn{4}{c}{ high DER production capacity } \\
\hline \multicolumn{1}{c||}{} & 0 & 500 & 1000 & 1500 & 0 & 500 & 1000 & 1500 \\
\hline \hline no storage & 2079.0 & 1683.1 & 1426.0 & 1255.5 & 1489.7 & 1054.1 & 723.92 & 440.09 \\
\hline with storage & 2062.2 & 1654.0 & 1387.5 & 1208.7 & 1467.0 & 1017.2 & 685.33 & 391.42
\end{tabular}

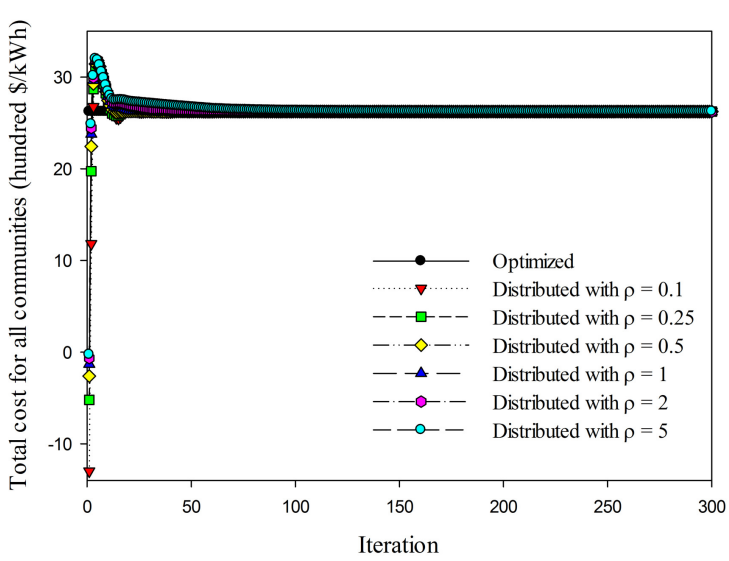

Fig. 6. Convergence of ADMM

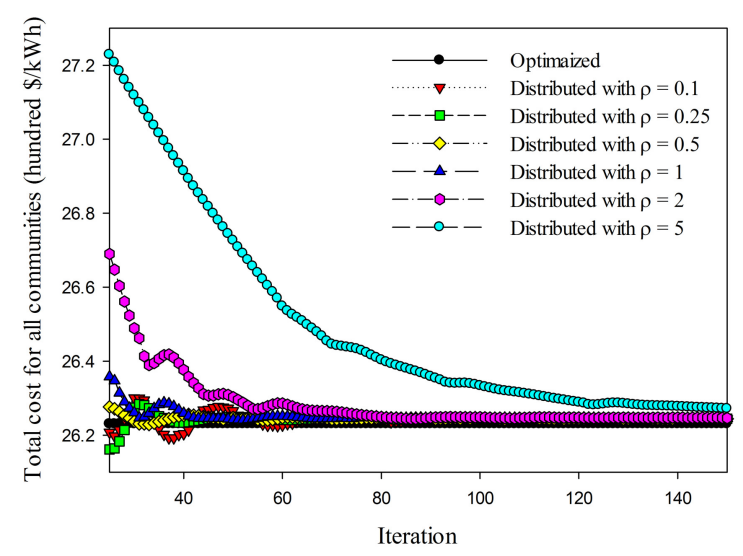

Fig. 7. Extracted iteration 25 to 150 from Fig. 6

in Fig. 3. Three communities are assumed to connect on the same power distribution line, and the threshold discussed in our previous work in [7] is set to 5 for the individual adjusting process for each community (IndiAdj). Each community may generates the inaccurate data with an uniform distribution, $\mathcal{U}(-25,25)$, with the probability of $\operatorname{Err}$ at every time step. Once the accumulated inaccurate data exceed the threshold, the communication will be initiated among communities and trigger the ADMM for the next round. That is, the count of communication package (CommPkg\#) will increase by 2 , and the count of performed ADMM (ADMM\#) will increase by 1 . The results with three different settings of the crosscommunity adjustment threshold (i.e., 2.5, 2.75 and 3 times the

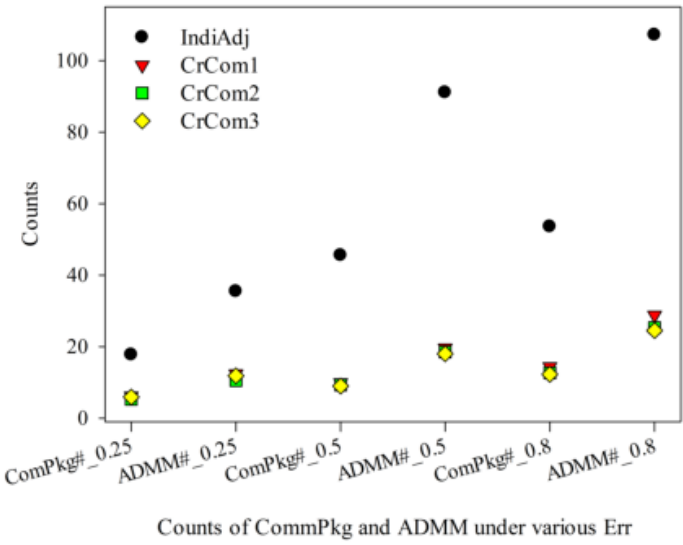

Fig. 8. Advantage of Cross-community adjustment

threshold of the single community as $C r C o m 1, C r C o m 2$, and $\mathrm{CrCom} 3$ respectively) are presented in Fig. 8. The number in the x-axis indicates the three cases with the probability of $\mathrm{Err}$ as $0.25,0.5$ and 0.8 . The counts in each case are the average of 10 experiment runs. The advantage of XCA is successfully shown with the increasing difference between the IndiAdj and other three $\mathrm{CrCom}$ scenarios. The difference becomes more significant when the probability of Err is larger.

\section{CONCLUSiON}

The cross-community collaboration (XCC) is proposed for the cloud-based energy management service in this paper. With the enabled cross-community collaboration among the involved communities, the energy management service can deliver an incentive to engage customers by minimizing global costs (including environmental cost and electricity cost). The proposed XCC also shows the potential for engaging customers without a storage system to the energy management service. The cloud-based energy management service with the $\mathrm{XCC}$ is formulated as the distributed multi-block ADMM problem to practically and efficiently handle the massive data and variables with various allocated cloud computing resources. The communication time for the distributed XCC is small as different cloud computing resources utilize it from different energy management service providers at the intercloud or the intra-cloud level. The cross-community adjustment (XCA) is also proposed to enhance the efficiency of XCC under uncertainty by reducing the unnecessarily overwhelmed data exchanging and computations. 


\section{REFERENCES}

[1] U.S. Department of Energy, "Consumer engagement." Available at https://www.smartgrid.gov/the_smart_grid/consumer_engagement.html, accessed 2021.

[2] P. Siano, "Demand response and smart grids?a survey," Renewable and Sustainable Energy Reviews, vol. 30, no. C, pp. 461-478, 2014. Available at https://EconPapers.repec.org/RePEc:eee:rensus:v:30:y:2014:i:c:p: 461-478.

[3] S. Maharjan, Q. Zhu, Y. Zhang, S. Gjessing, and T. Basar, "Dependable demand response management in the smart grid: A stackelberg game approach," IEEE Transactions on Smart Grid, vol. 4, pp. 120-132, March 2013.

[4] Netmetering. Available at http://en.wikipedia.org/wiki/Net_metering, accessed 2021.

[5] Retail Electric Providers Certification and Reporting, "Retail electric providers." Available at https://www.puc.texas.gov/industry/electric/ business/rep/Rep.aspx, accessed 2021.

[6] Green Mountain Energy, "Renewable rewards® buyback program," 2020.

[7] Y. Chen and J. M. Chang, "Emaas: Cloud-based energy management service for distributed renewable energy integration," IEEE Transactions on Smart Grid, vol. 6, no. 6, pp. 2816-2824, 2015.

[8] Y. Chen and J. M. Chang, "Fair demand response with electric vehicles for the cloud based energy management service," IEEE Transactions on Smart Grid, vol. 9, no. 1, pp. 458-468, 2018.

[9] Z. Tan, P. Yang, and A. Nehorai, "An optimal and distributed demand response strategy with electric vehicles in the smart grid," Smart Grid, IEEE Transactions on, vol. 5, no. 2, pp. 861-869, 2014.

[10] S. Boyd, N. Parikh, E. Chu, B. Peleato, and J. Eckstein, "Distributed optimization and statistical learning via the alternating direction method of multipliers," Foundations and Trends ${ }^{\circledR}$ in Machine Learning, vol. 3, no. 1, pp. 1-122, 2011.

[11] R. Zhang and J. Kwok, "Asynchronous distributed admm for consensus optimization," in Proceedings of the 31st International Conference on Machine Learning (ICML-14), pp. 1701-1709, 2014.

[12] M. Kraning, E. Chu, J. Lavaei, and S. Boyd, "Message passing for dynamic network energy management," arXiv preprint arXiv:1204.1106, 2012.

[13] J. Rivera, P. Wolfrum, S. Hirche, C. Goebel, and H.-A. Jacobsen, "Alternating direction method of multipliers for decentralized electric vehicle charging control," in Decision and Control (CDC), 2013 IEEE 52nd Annual Conference on, pp. 6960-6965, IEEE, 2013.

[14] Y. Zhang and G. Giannakis, "Efficient decentralized economic dispatch for microgrids with wind power integration," in Green Technologies Conference (GreenTech), 2014 Sixth Annual IEEE, pp. 7-12, IEEE, 2014.

[15] T.-H. Chang, M. Hong, and X. Wang, "Multi-agent distributed optimization via inexact consensus admm," Signal Processing, IEEE Transactions on, vol. 63, no. 2, pp. 482-497, 2015.

[16] L. Mei, W. K. Chan, and T. H. Tse, "A tale of clouds: Paradigm comparisons and some thoughts on research issues," in 2008 IEEE AsiaPacific Services Computing Conference, (Yilan, Taiwan), pp. 464-469, IEEE, Dec 2008.

[17] S. Chen, S. Nepal, and R. Liu, "Secure connectivity for intra-cloud and inter-cloud communication," in 2011 40th International Conference on Parallel Processing Workshops, (Taipei, Taiwan), pp. 154-159, IEEE, 2011.

[18] Public Utility Commission of Texas, "Electric substantive rule 25.211." Available at https://www.puc.texas.gov/agency/rulesnlaws/ subrules/electric/25.211/25.211.pdf, accessed 2015.

[19] W. Deng, M. Lai, Z. Peng, and W. Yin, "Parallel multi-block admm with o(1 / k) convergence," Journal of Scientific Computing, vol. 71, 11 2016.

[20] M. Hong, M. Razaviyayn, Z. Q. Luo, and J. S. Pang, "A unified algorithmic framework for block-structured optimization involving big data: With applications in machine learning and signal processing," IEEE Signal Processing Magazine, vol. 33, pp. 57-77, Jan 2016.

[21] California ISO. Available at http://www.caiso.com/Pages/default.aspx, accessed 2021.

[22] Tesla, Inc., "Tesla powerwall 2." Available at https://www.tesla.com/ powerwall, accessed 2021.

[23] D. Doughty, "Vehicle battery safety roadmap guidance," tech. rep., National Renewable Energy Laboratory (NREL), Golden, CO., 2012.
[24] J. Sun, V. Palade, X. Wu, W. Fang, and Z. Wang, "Solving the power economic dispatch problem with generator constraints by random drift particle swarm optimization," Industrial Informatics, IEEE Transactions on, vol. 10, pp. 222-232, Feb 2014. 